Dohi, Shinichi; Nakamura, Shogo; Sakurai, Yoshitaka;Tsuruta, Setsuo; Knauf, Rainer:

Dynamic learning need reflection system for academic education and its applicability to intelligent agents

Zuerst erschienen in:

Sixth IEEE International Conference on Advanced Learning Technologies : ICALT 2006 ; proceedings ; 5 - 7 July, 2006, Kerkrade, The Netherlands / sponsored by IEEE Technical Committee on Learning Technology ... - Los Alamitos, Calif. : IEEE Computer Society, c2006. - ISBN 0-7695-2632-2, S. 459-463 Referenz-Link IEEE: http://ieeexplore.ieee.org/xpls/abs all.jsp?tp=\&arnumber=1652471\&is number $=34637$ 


\title{
Dynamic Learning Need Reflection System for Academic Education and it's Applicability to Intelligent Agents
}

\author{
Yoshitaka Sakurai ${ }^{1}$, Shinichi Dohi ${ }^{1}$, Shogo Nakamura ${ }^{1}$, Setsuo Tsuruta $^{1}$, Rainer Knauf ${ }^{2}$ \\ ${ }^{1}$ Tokyo Denki University, Japan $\quad{ }^{2}$ Technical University Ilmenau, Germany \\ \{ysakurai,dohi,nakamura,tsuruta\}@sie.dendai.ac.jpｒainer.knauf@tu-ilmenau.de
}

\begin{abstract}
A new concept DLNR (Dynamic Learning Need Reflection) and its system used in the education at a university are suggested. The effects, particularly on the learning of software agents, are analyzed.

DLNR's goal is to increase students' learning motivation through dynamically clarifying and reflecting their learning need. To attain this, DLNR includes "prerequisite conditions", "no compulsory subjects", "payment for each subject" and "Grade Point Average: GPA" to estimate learning results.

By using a tool to realize DLNR, students design their learning need, namely their graduation timeline, by themselves to achieve their academic goal towards their job after graduation. Through taking classes, students dynamically modify the timeline reflectively according to the intermediate results shown by GPA.

DLNR's effects are evaluated. Particularly, DLNR was found applicable to the learning of software agents for intelligent system assistants, through incorporating more general tools such as Storyboard.
\end{abstract}

\section{Introduction}

The School of Information Environment (SIE) at Tokyo Denki University (TDU) launched its programs in 2001 and its Graduate course did in 2004. Its mission is to promote students' eagerness for the study of technology that helps create an effective information environment necessary in the society. The core educational features of SIE are based upon a new concept called Dynamic Learning Need Reflection (DLNR).

In recent years, it has been pointed out that the motivation of students' study has decreased on their entering universities. To cope with this, DLNR is introduced at SIE, and students create their own personal graduation timelines (personal class schedules until graduation) [9]. They set their own career goals for jobs after graduation and select subjects to attain them. Creating a graduation timeline has a significant effect on students' motivation [8].
However, it takes a lot of manpower and time for students to make graduation timelines by hand in complex educational system. Thus, a Dynamic Syllabus (DS) tool based on DLNR was developed [1].

There is a DS developed at Georgetown University in the US also [6]. This is an advanced type of online versions of paper syllabi. This is a list of links divided or classified into fields or disciplines each of which has the links to electronic resources for classes, exercises, virtual exhibitions, and so on. This DS is used by Curriculum developers to make course materials for each subject or, at most, project. Such developers are not students, and develop common curriculum.

Our DS differs in that it is used by individual students in planning their own personal curriculum which means their own career or their own graduation timelines to attain their academic goal towards jobs after graduation. Using our DS based on DLNR concept, their personal graduation timelines can be modified by themselves easily and reflectively through severe estimation by GPA system. The goal of DLNR concept is to clarify students' learning needs and increase their motivation.

First, the DLNR concept and its system including our DS tool are described. Next, the observed effects on practical education are discussed. Then, the effects and/or the applicability to the learning of software agents for intelligent system assistants are discussed and compared with other learning support concepts.

\section{New Educational Concept \& System}

\subsection{The New Concept of DLNR}

A significant characteristic of SIE is an educational method. This is a new experiment of university level in Japan. The new educational concept DLNR aims at encouraging students to increase their learning motivation, to develop the spirit of independence, to keep up with globalization, and to foster a lively imagination. Important features of DLNR are:

Abolition of the Academic Year : The academic 
year as usual at Japanese universities has been abolished. As there is no first or second academic year strictly combined with compulsory subjects, they can more easily graduate in 3 or 3.5 years.

Abolition of the Compulsory Subject : Conventional compulsory subjects have been abolished, while prerequisite conditions are introduced as a subject necessary to learn its advanced subjects. This way, students can learn various subjects systematically. There are two prerequisite condition types, (1) a subject required to learn before learning its advanced subjects and (2) a recommendation to do so.

Credit System : A unit-based tuition was introduced, i.e. students pay a fee corresponding to the number of units per subject. Thus, students don't take a subject without a real need or interest. Moreover, it encourages students to make the maximum effort to take the subject worthy of the invested money.

Class Period Lengths : In SIE, the traditional Japanese 90 min class is replaced by 50 or 75 min class. $50 \mathrm{~min}$ classes are held three times a week for $50 \mathrm{~min}$ per class while 75 min classes twice a week, rather than just once. As a result, it is expected that students can concentrate for the entire period of each class and remember what they learned at the previous class.

Table 1 Grade Point Values

\begin{tabular}{|l|c|}
\hline Rank & Point \\
\hline S $(90-100)$, A $(80-89)$ & 4 \\
\hline B ( 70-79) & 3 \\
\hline C (60-69) & 2 \\
\hline D ( 40-59), E $(0-39)$ & 0 \\
\hline
\end{tabular}

GPA System : The maximum number of units $n_{\max }$ students can take during one semester is determined by their GPA of the previous semester:

$$
n_{\max }=\left\{\begin{array}{lll}
25 & \text {, if } & G P A \geq 3 \\
12 & \text {, if } & G P A \leq 1
\end{array}\right.
$$

Thus, students have to reflect on the GPA and develop ideas to keep their GPA up. If they try to take too many units, it tends to decrease. If they try to raise their GPA in the semester, they have a tendency to take fewer units. The GPA is

$$
G P A=\frac{\sum_{i=1}^{n} u_{i} g_{i}}{\sum_{i=1}^{n} r_{i}}
$$

with $u_{i}$ being the number of units, $g_{i}$ being the grade points earned, and $r_{i}$ being the maximum number of units for course $i$. And $n$ is the total number of courses in the semester. Table 1 shows Grade Point values.

\subsection{Introduction to the SIE Program}

At SIE, all freshmen attend the Introduction to the SIE program consisting of two subjects, Curriculum
Planning Class and Work Shop. This aims at making all students understand SIE's philosophy such as DLNR concept. Students clarify their learning needs and improve their motivation by planning their own curriculum to attain their dreams and career goal.

\section{Curriculum Planning Class}

On entering the university, students create their own graduation timeline (students' personal curriculum or individual students' class schedules till their graduation). The completed graduation timeline including post-graduation goals is submitted to SIE. Though seemingly a daring plan, it is natural and important to make clear each student's target upon entering the university. Whenever the semester passed, the class schedules need to be updated. Thus, every time a semester begins, updated class schedules are submitted on the Internet. Students can connect to the Internet anytime and anywhere on campus until graduation without charge. As the result, classes are attended by students who have increased their motivation by clearly knowing their own articulated targets.

\subsection{Dynamic Syllabus (DS)}

It is challenging to make the timetable (timeline) by hand since the relationships among prerequisite conditions, GPA, and other aspects are complex. Then, we developed DS to assist students on the Internet [2][3][4][5]. DS architecture is shown in Fig. 1.

In order to help students decide on goals for their post-graduation, DS displays subjects systematically and assists to understand how to learn the subjects in a natural order. According to the conditions of SIE's education system, DS assesses the EDP (Electric Data Processing system) that provides information on students' results. After getting the students' information from EDP, students can make the class schedule, using DS that displays the syllabus with students' result information consistent with that of EDP.

\section{How to Make Class Schedule}

Fig. 2 shows how to make graduation timetables using DS. (1) Students select subjects from the table until graduation conditions are satisfied. The conditions of graduation include: $\geq 2$ units in Introduction to SIE Program and Computer Literacy, $\geq 40$ units in Cultural subjects, $\geq 60$ units in major subjects, $\geq 22$ units in other subjects, and $\geq 124$ units totally.

(2) Checking prerequisite conditions, a class schedule is made. (3) The class schedule is simulated for checking contradictions. (4) Subjects are registered only in the current semester. All class schedules from the first semester till graduation are created, repeating (2)-(4). 


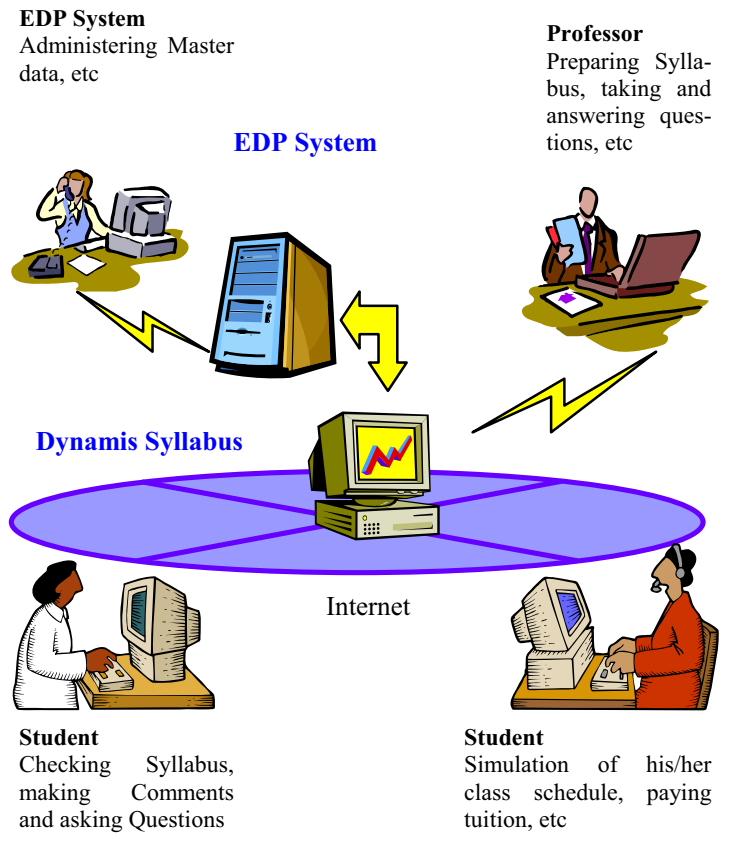

Fig. 1 Architecture of Dynamic Syllabus system (DS)

\section{Selection of Subject}

DS is accessed via Internet by the URL, clicking the menu item of Curriculum Planning, and student's login. Subjects are selected in a curriculum setup-window split into an upper (spring semester) and a lower (fall semester) part. Due to abolishing the academic year, there are only these two semesters.

\section{Information on Subject and Prerequisite Condition}

Each subject is listed along with the number of units (in parentheses), a bookmark, and/or a starmark (Fig. 3). Clicking the bookmark opens a syllabus window. Clicking the starmark displays the prerequisite condition. E.g., by clicking the red starmark of Computer Programming, the background color of the subjects whose prerequisite condition is Computer Programming, change to light purple color (Fig. 4).

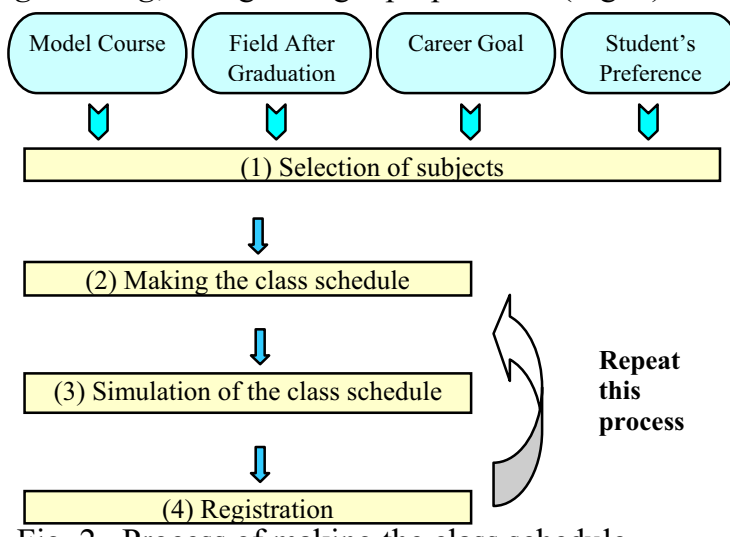

Fig. 2 Process of making the class schedule
Prototyping and Completing Class Schedule

After the subjects are selected, a prototype of class schedules is shown. As students click subjects they will attend at specific times in specific days of the week, the class schedule is generated. If errors occur, messages are displayed. After correction, the class schedule is completed.

\section{Computer Graphics [2.0] $\triangleq \star$}

Fig. 3 Information of Subject

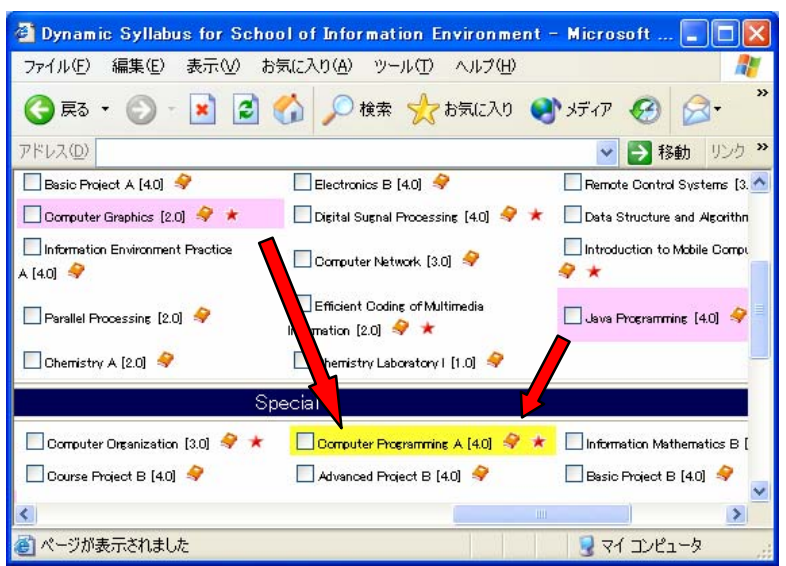

Fig. 4 Prerequisite condition

\section{Effects on Practical Education}

\subsection{Effects of the Curriculum Planning Class}

Effects of the DS for DLNR were investigated through a questionnaire in Curriculum Planning class. 203 students have been asked. The rate of students answering, it is useful to make class schedules until graduation, was around $80 \%$. It was more than $80 \%$ as to class schedules for only the current semester. These show the effect of DLNR and DS on increasing learning motivation.

As further results of this investigation, more than $50 \%$ of the students were able to create their class schedules in two hours. About $50 \%$ of the students, who could not complete their class schedule during this class (10 hours), were given a week to complete it as homework. Totally 162 out of $173(96.3 \%)$ students were finally able to complete their class schedules within less than 20 hours. These shows that the DS succeeded in making DLNR concept feasible and thus effective.

\subsection{Effects of the GPA and Credit System}

Two classes under the same conditions were compared, an undergraduate class taught in the new system 
based on DLNR and a postgraduate class taught in the old one. The former had no drop out, but in the latter, $50 \%$ of all students dropped out or gave up halfway. Both classes had a lot of homework anytime.

In the old system, they need not to pay for the class. Their GPA doesn't change if they don't have the examination, namely if they drop out. This shows severe but reasonable estimation such as GPA and credit system is effective. Students should clarify their learning need and be moderately controlled so that they can overcome the difficulties such as a heavy home work.

$30 \%$ of the remaining students in the old system couldn't get the credit. Meanwhile, less than 5\% in the new system failed. This suggests that a shorter class period (50 min vs. $150 \mathrm{~min}$ ) for concentration, and a higher frequency (3 times vs. once a week) for remember, are effective for learning.

\section{Applicability for Intelligent Agent's Learning}

\subsection{Intelligent Agents' Learning}

Learning takes much time and load. Meanwhile, Intelligent Software Agents (IAs) have to learn efficient rules, procedures, or knowledge in order for them to respond smartly. They not only acquire or mine it from data or experience but also maintain it whenever new knowledge comes up. Here, to maintain means to validate, refine (for consistency), generalize (for efficiency), or re-organize (for both). If they find unsolvable problems, they have to ask humans or other IAs. If too many such problems remain unsolved, they can't maintain the knowledge chunk related to the subject. They can't recognize what part of the chunk is right, and their learning motivation decreases.

Thus, IAs are similar to human students in learning. They have to divide knowledge into several chunks corresponding to subjects, and learn per subject as in practical education. Then, IAs can apply DLNR to emulate their learning/education as follows:

- No Academic Year: IAs are given no deadline or just essential dynamic deadlines instead of unnecessary fixed ones to learn a group of subjects.

- No Compulsory Subject: IAs choose learning needs (LNs) by themselves without given LN, using their goals, resources, and environment.

- Prerequisite Condition: IAs clarify or optimize LN through reflection on constraints.

- Credit System: IAs prioritize LNs (subjects), reflecting on effective use of a limited amount of (virtual) money supposed to be given to each IA.

- Short Class Period (50 min): For IAs to learn efficiently they requires interactive learning processes, where each block of consecutive interaction is shorter in period and occurs more frequently.

- GPA: IAs need severe systems for estimating their learning results step by step.

- Dynamic Syllabus: IAs need efficient software tools or libraries to facilitate or emulate DLNR.

- Curriculum Planning Class: IAs should be trained in choosing functions of tools and adjusting such parameters as influence GPA or Credit System.

\subsection{Comparison with Storyboards}

Storyboarding [7] aims at representing didactic or teaching knowledge. Meanwhile, DS focuses on representing and reflecting LNs. Storyboards have a multiple level tree structure of graphs, whose edges represent didactics. DS has a multiple level multiple stage linear structure whose instance represents each students' LN. The top level consists of ordered stages. Each stage represents a semester and can be considered a node in storyboarding for representing a set of subjects. Each subject is also a node and has links to prerequisite subjects. In a further nesting level, each subject has a link to so-called Syllabus to represent teaching items.

Though partly fixed or mixed into its system, DS includes didactic knowledge such as one controlling or modeling LN to realize DLNR concept. Storyboarding doesn't include such concrete knowledge. However, it is more general and flexible, and can represent such knowledge. To build a learning system of IAs, storyboarding is useful if didactic knowledge of DLNR and DS is incorporated there.

\subsection{Applicability to Intelligent Agents}

As is derived from 4.1, using DS as a simulator or emulator to realize DLNR Concept, IAs can also create their own individual learning schedules to clarify their LN through trial and error. As suggested by the Effect of the Curriculum Planning Class (see section 3), IAs should be trained how to use tools such as DS.

Learning goals corresponding to academic goals are system functions supported by IAs. And each learning subject is derived from sub-goals. Each learning subject is considered to correspond to each function or each chunk of knowledge of an IA. Constraints among each of system functions are represented as Prerequisite Conditions. These are mutual relationships and rather dynamic than those represented by such as compulsory subjects. Each IA's personal curriculum is created through allocating each learning subjects in the time axis, considering prerequisite condition and other conditions.

Estimation methods such as GPA and estimation timing such as a semester should also be considered. 
On the same estimation timing, all subjects or LNs of each IA are triggered to synchronously modify the learning schedule, since they relate to each other by prerequisite conditions and so on. At each estimation timing, learning schedules are dynamically modified, reflecting intermediate learning results such as GPA and its related problems such as the unsatisfaction of prerequisite condition and change of goal themselves or various problems encountered while learning. As a result, the LNs can be clarified, dynamically reflecting on their goals, resources including their competence, and environments. Thus, IAs can learn efficiently and dependably towards their learning goal through such dynamic reflection.

The effect of the GPA and Credit System (see section 3) teaches that, through introducing severe but reasonable estimations, IAs can also be easily controlled like human to achieve their (sub) goals namely to dynamically clarify and satisfy their LNs.

As to learning goals, DLNR and DS are restricted to academic goals. Meanwhile, IAs should learn as long as they live. This is life learning in case of humans.

Whenever their functional requirements increase, IAs will be given new learning goals. Through deriving a set of learning subjects or LNs from added or modified requirements, DLNR is possibly used also in the learning or education of IAs as in an academic education of human students.

\section{Conclusion}

DLNR (Dynamic Learning Need Reflection) Concept and its system including a tool DS (Dynamic Syllabus) were proposed. Its effects in practical education were discussed. The effects and the applicability to learning of Intelligent Software Agents (IAs) were discussed and compared with those of didactic knowledge representation systems such as storyboarding.

As a result, it was shown that, due to DLNR and its tool DS, students can increase their learning motivation through clarifying and dynamically reflecting their LN. Furthermore, DLNR was found applicable to the learning of IAs through incorporating its concept or knowledge to tools such as a storyboard [7].

\section{Acknowledgement}

Authors gratefully acknowledge the contributions of Takamasa Shimada, Takashi Kohama, Masaki Kawakatsu and Klaus P. Jantke for DS and storyboarding.

\section{References}

[1] Dohi, S. \& Nakamura, S. \& Shimada, T. \& Kawakatsu, M., "The Development of the Dynamic Syllabus", JSEE, 2001, pp. 21-24.

[2] Dohi, S. \& Nakamura, S. \& Shimada, T. \& Kawakatsu, M., "The Introduction of the Dynamic Syllabus", CIEC, 2001, pp. 40-41.

[3] Dohi, S. \& Nakamura, S. \& Shimada, T. \& Kawakatsu, M., "Rising in the sense of purpose by introduction of The Dynamic Syllabus", IPSJ, no4, 2001, pp. 195-196.

[4] Dohi, S. \& Nakamura, S. \& Shimada, T. \& Kawakatsu, M., "Using of The Dynamic Syllabus in introduction to course", EIP2K, 2001, pp. 275-278.

[5] Dohi, S. \& Kohama, T. \& Nakamura, S., "Improvement plan of The Dynamic Syllabus", IPSJ, no4, 2002, pp. 253254

[6] Georgetown University, Dynamic Syllabus, http://www.georgetown.edu/crossroads/webcourses.html, 2006.

[7] Jantke, K.P. \& Knauf, R., "Didactic Design though Storyboarding: Standard Concepts for Standard Tools", Proc. $4^{\text {th }}$ Intl. Symposium on Information \& Communication Technologies, Cape Town, South Africa, 2005, New York: ACM Press, ISBN 0-9544145-6-X, 2005, pp. 20-25.

[8] SIE., The student handbook, School of Information Environment Tokyo Denki University, 2002.

[9] SIE., School of Information Environment Tokyo Denki University, http://www.sie.dendai.ac.jp/, 2006 